Mathematical Research Letters 3, 231-239 (1996)

\title{
ABSOLUTE CONTINUITY OF BERNOULLI CONVOLUTIONS, A SIMPLE PROOF
}

\author{
Yuval Peres AND Boris Solomyak
}

\begin{abstract}
A вstract. The distribution $\nu_{\lambda}$ of the random series $\sum \pm \lambda^{n}$ has been studied by many authors since the two seminal papers by Erdös in 1939 and 1940. Works of Alexander and Yorke, Przytycki and Urbański, and Ledrappier showed the importance of these distributions in several problems in dynamical systems and Hausdorff dimension estimation. Recently the second author proved a conjecture made by Garsia in 1962, that $\nu_{\lambda}$ is absolutely continuous for a.e. $\lambda \in(1 / 2,1)$. Here we give a considerably simplified proof of this theorem, using differentiation of measures instead of Fourier transform methods. This technique is better suited to analyze more general random power series.
\end{abstract}

\section{Introduction}

Consider the random series

$$
Y_{\lambda}=\sum_{n=0}^{\infty} \pm \lambda^{n}, \quad \text { for } 0<\lambda<1,
$$

where the "+" and "-" signs are chosen independently with probability $1 / 2$. Let $\nu_{\lambda}$ be the distribution of $Y_{\lambda}$ :

$$
\nu_{\lambda}(E)=\operatorname{Prob}\left\{Y_{\lambda} \in E\right\} .
$$

The measure $\nu_{\lambda}$ is the infinite convolution product of $\frac{1}{2}\left(\delta_{-\lambda^{n}}+\delta_{\lambda^{n}}\right)$ for $n \geq 0$ and is sometimes called an "infinite Bernoulli convolution". The Fourier transform of $\nu_{\lambda}$ can be represented as a convergent infinite product: $\hat{\nu}_{\lambda}(u)=\prod_{n=0}^{\infty} \cos \left(\lambda^{n} u\right)$.

Infinite Bernoulli convolutions have been studied since the 1930's; we sketch some of the relevant background below. The following theorem was recently proved by the second author, who verified a conjecture of Garsia (1962). Our goal here is to present a self-contained simple proof of this result.

Received December 10, 1995.

1991 Mathematics Subject Classification. Primary 28A12, Secondary 42A61, 60G50.

The authors were supported in part by NSF grants DMS-9404391 and DMS-9500744. 
Theorem 1 [Solomyak (1995)]. For a.e. $\lambda \in\left(\frac{1}{2}, 1\right)$ the measure $\nu_{\lambda}$ is absolutely continuous and has an $L^{2}$-density.

The main simplification in the present proof is the replacement of the Fourier transform estimates in Solomyak (1995) by the differentiation method used by Mattila (1990) to prove projection theorems. This method is more powerful and yields some extensions of Theorem 1 (see Section 4). Heuristically, the measures $\nu_{\lambda}$ may be viewed as "nonlinear projections" of the uniform measure on sequence space; the nonlinearity is controlled by establishing a transversality property of certain power series. This property is closely related to the bounds on double zeros used in the original proof, but is easier to verify.

Our new proof of Theorem 1 is in sections $2-3$. We now describe some of the previous work on infinite Bernoulli convolutions. Jessen and Wintner (1935) showed that $\nu_{\lambda}$ is either absolutely continuous or purely singular, depending on $\lambda$; see Breiman (1968) for a proof using Kolmogorov's 01 Law. Kershner and Wintner (1935) observed that $\nu_{\lambda}$ is singular for $\lambda \in\left(0, \frac{1}{2}\right)$, since it is supported on a Cantor set of zero Lebesgue measure. Wintner (1935) noted that $\nu_{\lambda}$ is uniform on $[-2,2]$ for $\lambda=1 / 2$, and for $\lambda=2^{-1 / k}$ with $k \geq 1$ it is absolutely continuous, with a density having $k-1$ derivatives.

For any $\lambda \in(0,1)$, the closed support $K_{\lambda}$ of $\nu_{\lambda}$ satisfies the equation

$$
K_{\lambda}=\left(1+\lambda K_{\lambda}\right) \cup\left(-1+\lambda K_{\lambda}\right) .
$$

It is easy to show that there exists a unique compact set $K_{\lambda} \subset \mathbb{R}$ for which this equation holds (see, e.g. Hutchinson (1981)). If $\lambda \in\left(\frac{1}{2}, 1\right)$, then the interval $\left[-(1-\lambda)^{-1},(1-\lambda)^{-1}\right]$ satisfies the same equation, and therefore it must coincide with $K_{\lambda}$. Thus one might surmise that $\nu_{\lambda}$ is absolutely continuous for all such $\lambda$. However, by examining the Fourier Transform, Erdös (1939) showed that $\nu_{\lambda}$ is singular when $\lambda$ is the reciprocal of a PV (Pisot-Vijayaraghavan) number. Recall that a PV-number is an algebraic integer, all of whose conjugates are less than one in modulus; the golden ratio $(1+\sqrt{5}) / 2$ is the best known example. No other $\lambda \in\left(\frac{1}{2}, 1\right)$ are known to give singular $\nu_{\lambda}$.

In another direction, Erdős (1940) showed that $\nu_{\lambda}$ is absolutely continuous for a.e. $\lambda \in(a, 1)$ for some $a<1$ (in his proof $a$ is rather close to 1 and not given explicitly). Examples of algebraic $\lambda \in\left(\frac{1}{2}, 1\right)$ (other than $2^{-1 / k}$ ) with absolutely continuous $\nu_{\lambda}$ were found by Garsia (1962). In the same paper, Garsia conjectured that absolute continuity holds for a.e. $\lambda$ in the full interval $\left(\frac{1}{2}, 1\right)$. Kahane and Salem (1958) obtained a criterion for the measure $\nu_{\lambda}$ to have $L^{2}$-density, but until recently it was not clear how 
it could be applied. Applications of absolute continuity of $\nu_{\lambda}$ to dynamical systems and Hausdorff dimension were given by Alexander and Yorke (1984), Przytycki and Urbański (1989), and Ledrappier (1992).

\section{Beginning of the proof}

Let $B_{r}(x)=[x-r, x+r]$. Consider the lower derivative of $\nu_{\lambda}$ :

$$
\underline{D}\left(\nu_{\lambda}, x\right)=\liminf _{r \downarrow 0}(2 r)^{-1} \nu_{\lambda}\left[B_{r}(x)\right] .
$$

A standard application of the Vitali covering theorem shows that $\nu_{\lambda}$ is absolutely continuous if and only if $\underline{D}\left(\nu_{\lambda}, x\right)<\infty$ for $\nu_{\lambda}$ almost all $x \in \mathbb{R}$ (see, e.g. Mattila (1995) §2.12). If we show that

$$
\mathcal{S}:=\int_{I} \int_{\mathbb{R}} \underline{D}\left(\nu_{\lambda}, x\right) d \nu_{\lambda}(x) d \lambda<\infty
$$

then this criterion yields that $\nu_{\lambda}$ is absolutely continuous for a.e. $\lambda \in I$. The interval $I$ will be specified later; see the last section for measurability. Notice that for $\lambda$ such that $\nu_{\lambda}$ is absolutely continuous, $\underline{D}\left(\nu_{\lambda}, x\right)$ is a version of the Radon-Nikodym derivative $\frac{d \nu_{\lambda}(x)}{d x}$, so $\int_{\mathbb{R}} \underline{D}\left(\nu_{\lambda}, x\right) d \nu_{\lambda}(x)<\infty$ implies that $\frac{d \nu_{\lambda}(x)}{d x} \in L^{2}(\mathbb{R})$. By Fatou's Lemma,

$$
\mathcal{S} \leq \liminf _{r \downarrow 0}(2 r)^{-1} \int_{I} \int_{\mathbb{R}} \nu_{\lambda}\left[B_{r}(x)\right] d \nu_{\lambda}(x) d \lambda .
$$

We need to restate the definition of $\nu_{\lambda}$ as the distribution of the random series $Y_{\lambda}$. Let $\Omega=\{-1,1\}^{\mathbb{Z}_{+}}$be the sequence space equipped with the product topology. Let $\mu$ be the Bernoulli measure on $\Omega$ with the weights $(1 / 2,1 / 2)$. There is a natural map $\Pi_{\lambda}: \Omega \rightarrow \mathbb{R}$, given by

$$
\Pi_{\lambda}(\omega)=\sum_{n=0}^{\infty} \omega_{n} \lambda^{n} .
$$

A moment's reflection gives

$$
\nu_{\lambda}=\mu \circ \Pi_{\lambda}^{-1}
$$

Now $\Pi_{\lambda}$ is continuous so we can change variables in (2):

$$
\mathcal{S} \leq \liminf _{r \downarrow 0}(2 r)^{-1} \int_{I} \int_{\Omega} \nu_{\lambda}\left[B_{r}\left(\Pi_{\lambda}(\omega)\right)\right] d \mu(\omega) d \lambda .
$$


Next, denote by $1_{A}$ the characteristic function of a set $A$ and use (3) again:

$$
\nu_{\lambda}\left[B_{r}\left(\Pi_{\lambda}(\omega)\right)\right]=\int_{\mathbb{R}} 1_{B_{r}\left(\Pi_{\lambda}(\omega)\right)} d \nu_{\lambda}=\int_{\Omega} 1_{\left\{\tau \in \Omega:\left|\Pi_{\lambda}(\tau)-\Pi_{\lambda}(\omega)\right| \leq r\right\}} d \mu(\tau) .
$$

Substitute this into (4), exchange the order of integration, and integrate with respect to $\lambda$ to obtain

$(5) \mathcal{S} \leq \liminf _{r \downarrow 0}(2 r)^{-1} \int_{\Omega} \int_{\Omega} \mathcal{L}\left\{\lambda \in I:\left|\Pi_{\lambda}(\tau)-\Pi_{\lambda}(\omega)\right| \leq r\right\} d \mu(\tau) d \mu(\omega)$,

where $\mathcal{L}$ is Lebesgue measure. Let

$$
\phi_{\tau, \omega}(\lambda)=\Pi_{\lambda}(\tau)-\Pi_{\lambda}(\omega)=\sum_{n=0}^{\infty}\left(\tau_{n}-\omega_{n}\right) \lambda^{n} .
$$

Notice that $\tau_{n}-\omega_{n} \in\{-2,0,2\}$. We need to estimate $\mathcal{L}\left\{\lambda \in I:\left|\phi_{\tau, \omega}(\lambda)\right| \leq\right.$ $r\}$. We can write

$$
\phi_{\tau, \omega}(\lambda)=2 \lambda^{k} g(\lambda)
$$

where $k=|\omega \wedge \tau|=\min \left\{n: \omega_{n} \neq \tau_{n}\right\}$ and $g$ is a power series with constant term \pm 1 . Without loss of generality, $\omega_{k}<\tau_{k}$ so that

$$
g(x)=1+\sum_{n=1}^{\infty} b_{n} x^{n}, \quad \text { with } \quad b_{n} \in\{-1,0,1\} .
$$

In order to estimate the integrand in (5), assume that on the interval $I=$ $\left[\lambda_{0}, \lambda_{1}\right]$ the following $\delta$-transversality condition holds for some $\delta>0$ :

(8) For any $g$ of the form (7) and any $x \in I$, if $g(x)<\delta$ then $g^{\prime}(x)<-\delta$.

The assumption means that the graph of each $g$ as in (7) crosses transversally, with slope at most $-\delta$, all horizontal lines below height $\delta$ that it meets. In the next section we will find intervals where (8) holds. We claim that this assumption implies that for all $g$ of the form (7) and all $\rho>0$,

$$
\mathcal{L}\{\lambda \in I:|g(\lambda)| \leq \rho\} \leq 2 \delta^{-1} \rho .
$$

This is obvious if $\rho \geq \delta$; otherwise, $g^{\prime}(\lambda)<-\delta$ whenever $|g(\lambda)| \leq \rho$ by $\delta$-transversality. Thus $g$ is monotone decreasing on the set in (9) with $\left|g^{\prime}\right|>\delta$, so the inequality (9) is proved. 
By (6), $\left|\phi_{\tau, \omega}(\lambda)\right| \leq r$ implies that $|g(\lambda)| \leq \lambda_{0}^{-k} r / 2$ for $\lambda \in I=\left[\lambda_{0}, \lambda_{1}\right]$. Applying (9) with $\rho=\lambda_{0}^{-k} r / 2$, we obtain

$$
\mathcal{L}\left\{\lambda \in I:\left|\phi_{\tau, \omega}(\lambda)\right| \leq r\right\} \leq \delta^{-1} \lambda_{0}^{-k} r .
$$

Substituting this in (5) yields

$$
\begin{aligned}
\mathcal{S} & \leq \delta^{-1} \liminf _{r \downarrow 0}(2 r)^{-1} \int_{\Omega} \int_{\Omega} \lambda_{0}^{-|\omega \wedge \tau|} r d \mu(\tau) d \mu(\omega) \\
& =(2 \delta)^{-1} \sum_{k=0}^{\infty} \lambda_{0}^{-k}(\mu \times \mu)\{(\omega, \tau):|\omega \wedge \tau|=k\} \\
& =(2 \delta)^{-1} \sum_{k=0}^{\infty} \lambda_{0}^{-k} 2^{-k-1}<\infty
\end{aligned}
$$

where the last step requires that $\lambda_{0}>\frac{1}{2}$. This proves (1), so we have shown the absolute continuity of $\nu_{\lambda}$ for a.e. $\lambda \in I$ assuming $\delta$-transversality.

\section{Establishing transversality, and conclusion of the proof}

Definition. A power series $h(x)$ is called a $(*)$-function if for some $k \geq 1$ and $a_{k} \in[-1,1]$,

$$
h(x)=1-\sum_{i=1}^{k-1} x^{i}+a_{k} x^{k}+\sum_{i=k+1}^{\infty} x^{i} .
$$

In Solomyak (1995) it was shown that among all convex combinations of series of the form (7), the power series with the smallest double zero must be a (*)-function. Here we bypass this optimization argument and prove directly that an estimate on a $(*)$-function and its derivative yield $\delta$-transversality.

Lemma. Suppose that a (*)-function $h$ satisfies

$$
h\left(x_{0}\right)>\delta \quad \text { and } \quad h^{\prime}\left(x_{0}\right)<-\delta
$$

for some $x_{0} \in(0,1)$ and $\delta \in(0,1)$. Then the transversality condition (8) holds on $\left[0, x_{0}\right]$.

Proof. First observe that $h(x)>\delta$ and $h^{\prime}(x)<-\delta$ for all $x \in\left[0, x_{0}\right]$. Indeed, $h^{\prime \prime}$ has at most one zero on $(0,1)$ being a power series with at most one coefficient sign change. We have $h^{\prime}(0)=-1$ if $k>1$ and $h^{\prime}(0)=a_{1}$ otherwise. Thus $h^{\prime}(0)<-\delta$ (if $k=1$ this follows from $h^{\prime}\left(x_{0}\right)<-\delta$ ). Since 
$h^{\prime}(1-)=+\infty$, we must have $h^{\prime}(x)<-\delta$ for all $x \in\left(0, x_{0}\right)$, otherwise $h^{\prime \prime}$ would have at least two zeros. Now obviously, $h(x)>h\left(x_{0}\right)>\delta$ for $x \in\left(0, x_{0}\right)$.

Let $g(x)$ be a power series of the form (7). Consider $f(x)=g(x)-h(x)$. Then (7) and the definition of a (*)-function imply that $f(x)=\sum_{i=1}^{l} c_{i} x^{i}-$ $\sum_{i=l+1}^{\infty} c_{i} x^{i}$, where $c_{i} \geq 0$ and $l=k-1$ or $l=k$. We have for any $x \in\left[0, x_{0}\right]$, by the claim proved above:

$$
g(x)<\delta \Rightarrow f(x)<0 \Rightarrow f^{\prime}(x)<0 \Rightarrow g^{\prime}(x)<-\delta .
$$

The middle implication is a consequence of one coefficient sign change:

$$
\begin{aligned}
f(x)<0 \Rightarrow & \sum_{i=1}^{l} c_{i} x^{i}<\sum_{i=l+1}^{\infty} c_{i} x^{i} \Rightarrow \\
& \sum_{i=1}^{l} c_{i} i x^{i-1}<\sum_{i=l+1}^{\infty} c_{i} i x^{i-1} \Rightarrow f^{\prime}(x)<0 .
\end{aligned}
$$

Proof of Theorem 1 concluded: Observe that if $\nu_{\lambda^{2}}$ is absolutely continuous, then so is $\nu_{\lambda}$, since it is a convolution of $\nu_{\lambda^{2}}$ with some other measure. Thus, it is enough to prove absolute continuity of $\nu_{\lambda}$ for a.e. $\lambda \in\left[\frac{1}{2}, 2^{-1 / 2}\right]$. First we establish that $\delta$-transversality holds in the smaller interval $\left[\frac{1}{2}, 2^{-2 / 3}\right]$, and then indicate how to cover $\left[2^{-2 / 3}, 2^{-1 / 2}\right]$.

In order to apply the lemma, it remains to find an appropriate $(*)$ function. Let

$$
h(x)=1-x-x^{2}-x^{3}+0.5 x^{4}+\sum_{i=5}^{\infty} x^{i}
$$

(found by a simple computer search). This is a (*)-function, satisfying $h\left(2^{-2 / 3}\right)>0.07$ and $h^{\prime}\left(2^{-2 / 3}\right)<-0.09$, so $\delta$-transversality is verified for the interval $\left[0,2^{-2 / 3}\right]$. The argument in Section 2 can be applied in $I=$ $\left[\lambda_{0}, 2^{-2 / 3}\right]$ for any $\lambda_{0}>\frac{1}{2}$, and this establishes absolute continuity for a.e. $\lambda \in\left[\frac{1}{2}, 2^{-2 / 3}\right]$.

Unfortunately, in this way we cannot cover $\left[\frac{1}{2}, 2^{-1 / 2}\right]$ since there is a power series of the form (7) with a double zero around 0.68 (see Solomyak (1995)), and having a double zero obviously contradicts $\delta$-transversality. In order to cover the interval $\left[2^{-2 / 3}, 2^{-1 / 2}\right]$ consider the "thinned" random series $Z_{\lambda}=\sum_{i \neq 2+3 j} \pm \lambda^{i}$, with every third term removed. If the distribution of $Z_{\lambda}$ is absolutely continuous, then so is $\nu_{\lambda}$. Replacing the series $Y_{\lambda}$ 
by $Z_{\lambda}$ is equivalent to replacing the uniform measure $\mu$ on $\Omega$ by a measure $\widetilde{\mu}$ on $\{-1,0,1\}^{\mathbb{Z}_{+}}$according to which every third symbol is forced to be 0 and all other symbols are -1 or 1 with probability $1 / 2$.

The analogue of (10) with $\widetilde{\mu}$ instead of $\mu$ goes through provided that $\lambda_{0}>2^{-2 / 3}$, since the $\widetilde{\mu} \times \widetilde{\mu}$ probability of having two sequences coincide up to the $k$-th term is $\sim 2^{-2 k / 3}$. Again, $\delta$-transversality is needed but for a smaller class, namely, for power series of the form (7) that satisfy either $b_{3 j+1}=0$ for all $j \geq 0$, or $b_{3 j+2}=0$ for all $j \geq 0$. We claim that in both cases $\delta$-transversality holds on $\left(0,2^{-1 / 2}\right)$. Indeed, the lemma has an obvious "thinned" version, and it suffices to check that the following two thinned (*)-functions

$$
\begin{aligned}
& h_{1}(x)=1-x-x^{3}-x^{4}+\sum_{j=2}^{\infty}\left(x^{3 j}+x^{3 j+1}\right), \\
& h_{2}(x)=1-x^{2}-x^{3}-x^{5}-x^{6}+\sum_{j=3}^{\infty}\left(x^{3 j-1}+x^{3 j}\right),
\end{aligned}
$$

satisfy the condition (11) at $x_{0}=2^{-1 / 2}$.

\section{Concluding remarks}

1. Strictly speaking, one needs to verify that the inner integral in (1) is measurable as a function of $\lambda$, before applying Fatou's Lemma. Using (3) this inner integral can be written as

$$
\int_{\Omega} \liminf _{r \downarrow 0}(2 r)^{-1} \int_{\Omega} 1_{\left\{\tau \in \Omega:\left|\Pi_{\lambda}(\tau)-\Pi_{\lambda}(\omega)\right| \leq r\right\}} d \mu(\tau) d \mu(\omega),
$$

and checking measurability in $\lambda$ of the last expression is routine.

2. In the first part of the proof of Theorem 1, the criterion of Kahane and Salem (1958) could be used instead of Mattila's approach used here.

3. We learned the idea of using transversality from Pollicot and Simon (1995).

4. It is natural to ask about the distribution of the random sum $Y_{\lambda}$ when the signs are governed by a nonuniform probability measure $\widetilde{\mu}$ on the sequence space $\Omega$, instead of the uniform measure $\mu$. The argument in section 2 is quite general, and shows that if the series (10) (with $\widetilde{\mu}$ in place of $\mu$ ) converges, then the corresponding distribution $\widetilde{\nu}_{\lambda}$ has $L^{2}$-density for a.e. $\lambda$ in any interval $I=\left[\lambda_{0}, \lambda_{1}\right]$ where the $\delta$-transversality condition holds. This result is sharp in the sense that if the series (10) with $\tilde{\mu}$ diverges, then for all $\lambda<\lambda_{0}$ the measure $\widetilde{\nu}_{\lambda}$ cannot have an $L^{2}$-density. In Peres and 
Solomyak (1995), analogous conditions are given for $\widetilde{\nu}_{\lambda}$ to have a density in $L^{q}$, with $q \in(1,2)$. In general, the critical $\lambda$ for different $q$ are distinct, and depend on the multifractal spectrum of $\widetilde{\mu}$. We illustrate these results in the case where $\widetilde{\mu}$ is Bernoulli, which corresponds to the random series $Y_{\lambda}$ with the signs chosen independently with probabilities $(p, 1-p)$. Let $\nu_{\lambda}^{p}$ be its distribution.

Theorem 2. Let $I$ be an interval where the $\delta$-transversality condition holds.

(a) $\nu_{\lambda}^{p}$ is singular for all $\lambda<p^{p}(1-p)^{1-p}$ and absolutely continuous for a.e. $\lambda>p^{p}(1-p)^{1-p}$ in the interval $I$.

(b) Let $q \in(1,2]$. The measure $\nu_{\lambda}^{p}$ does not have a density in $L^{q}$ for all $\lambda<\left(p^{q}+(1-p)^{q}\right)^{\frac{1}{q-1}}$, and has a density in $L^{q}$ for a.e. $\lambda>\left(p^{q}+(1-p)^{q}\right)^{\frac{1}{q-1}}$ in the interval $I$.

Mark Pollicott has informed us that Charmaine Leech, a graduate student at Warwick, has also been working in this direction.

5. As noted in Solomyak (1995), Theorem 1 immediately implies that for a.e. $\lambda \in\left[2^{-1 / 2}, 1\right]$, the density of $\nu_{\lambda}$ is continuous. It is unknown whether this also holds for a.e. $\lambda \in\left[\frac{1}{2}, 2^{-1 / 2}\right]$.

6. The absolute continuity of $\nu_{\lambda}$ in Theorem 1 is proven for a.e. $\lambda \in$ $\left[\frac{1}{2}, 1\right]$. What can be said about the exceptional set? The known exceptions, the reciprocals of PV-numbers, form a countable set which is known to be closed and have the maximal (isolated) point $\approx 0.7548777$. Salem (1943) proved the following converse to a result of Erdös (1939): If $1 / \lambda$ is not a PV-number then $\hat{\nu}_{\lambda}(u) \rightarrow 0$ as $u \rightarrow \infty$. As shown by Kahane (1971), the argument of Erdős (1940) implies that the Hausdorff dimension of the exceptional set in $(a, 1]$ tends to 0 as $a \rightarrow 1$. Perhaps a modification of our methods can give a bound on this Hausdorff dimension for all $a$, by analogy with a projection theorem of Falconer (1982).

\section{References}

1. J. C. Alexander and J. A. Yorke, Fat baker's transformations, Ergodic Th. Dyn. Syst. 4 (1984), 1-23.

2. L. Breiman, Probability, Addison-Wesley, 1968.

3. P. Erdős, On a family of symmetric Bernoulli convolutions, Amer. J. Math. 61 (1939), 974-975.

4. 62 (1940), 180-186.

5. K. Falconer, Hausdorff dimension and the exceptional set of projections, Mathematika 29 (1982), 109-115.

6. A. M. Garsia, Arithmetic properties of Bernoulli convolutions, Trans. Amer. Math. Soc. 102 (1962), 409-432. 
7. J. E. Hutchinson, Fractals and self-similarity, Indiana University Math. J. 30 (1981), 713-747.

8. B. Jessen and A. Wintner, Distribution functions and the Riemann zeta function, Trans. Amer. Math. Soc. 38 (1935), 48-88.

9. J. P. Kahane, Sur la distribution de certaines series aleatoires, Colloque Th. Nombres [1969, Bordeaux], Bull. Soc. math. France, Mémoire 25 (1971), 119-122.

10. J. P. Kahane and R. Salem, Sur la convolution d'une infinité de distributions de Bernoulli, Colloq. Math. 6 (1958), 193-202.

11. R. Kershner and A. Wintner, On symmetric Bernoulli convolutions, Amer. J. Math. 57 (1935), 541-548.

12. F. Ledrappier, On the dimension of some graphs, Contemporary Math. 135 (1992), 285-293.

13. P. Mattila, Orthogonal projections, Riesz capacities and Minkowski content, Indiana University Math. J. 39 (1990), 185-198.

14. Geometry of sets and measures in Euclidean spaces, Cambridge Universty Press, 1995.

15. Y. Peres and B. Solomyak, Self-similar measures and intersections of Cantor sets, preprint (1995).

16. M. Pollicott and K. Simon, The Hausdorff dimension of $\lambda$-expansions with deleted digits, Trans. Amer. Math. Soc. 347 (1995), 967-983.

17. F. Przytycki and M. Urbański, On Hausdorff dimension of some fractal sets, Studia Math. 93 (1989), 155-186.

18. R. Salem, On sets of uniqueness and sets of multiplicity, Trans. Amer. Math. Soc. 54 (1943), 218-228.

19. B. Solomyak, On the random series $\sum \pm \lambda^{i}$ (an Erdös problem), Ann. of Math. 142 (1995), 611-625.

20. A. Wintner, On convergent Poisson convolutions, Amer. J. Math. 57 (1935), 827838.

Department of Statistics, Univerity of California, Berkeley, Ca .

Current address: Institute of Mathematics, The Hebrew University, Jerusalem, Israel E-mail address: peres@math.huji.ac.il

Department of M athematics, Univerity of Washington, Seatthe, Wh $98195-4350$

E-mail address: solomyak@math.washington.edu 